\title{
Maxwell-Chern-Simons Models: Their Symmetries, Exact Solutions and Non-relativistic Limits
}

\author{
J. Niederle, A. G. Nikitin, O. Kuriksha
}

\begin{abstract}
Two Maxwell-Chern-Simons (MCS) models in the $(1+3)$-dimensional space-space are discussed and families of their exact solutions are found. In contrast to the Carroll-Field-Jackiw (CFE) model [2] these systems are relativistically invariant and include the CFJ model as a particular sector.

Using the Inönü-Wigner contraction a Galilei-invariant non-relativistic limit of the systems is found, which makes possible to find a Galilean formulation of the CFJ model.
\end{abstract}

\section{Introduction}

There are three motivations of the present paper. First we search for four-dimensional formulations of Maxwell-Chern-Simon models [1]. Secondly, we look for relativistic- and Galilei-invariant versions of the Carroll-Field-Jackiw electrodynamics [2]. At the third place we construct a relativistic counterpart of the Galilei-invariant equations for vector fields proposed in our paper [3].

There are two symmetries of Maxwell's electrodynamics that have dominated all fundamental physical theories, namely, gauge and Lorentz invariance. They provide physical principles that guide the invention of models describing fundamental phenomena. At the first place, the properties of the electromagnetic radiation (in a natural setting and in $\mathrm{HE}$ accelerators) are described by Lorentz-invariant dynamics. On the other hand, the gauge invariance is possible for massless field only, and so it is validated by the stringent limits on the photon mass $m_{\gamma}$.

Possible breaking of the Lorentz and gauge invariance has been tested within a theoretical framework with symmetry breaking parameters. The violation of gauge invariance was tested in frames of two modifications of the Maxwell theory. In the first of them the Lagrangian of the free e.m. field $\mathcal{L}_{E M}=-F_{\mu \nu} F^{\mu \nu}$ is modified to

$$
\mathcal{L}_{E M} \rightarrow \mathcal{L}_{E M}+\frac{m_{\gamma}^{2}}{2} A_{\nu} A^{\nu}
$$

where $F_{\mu \nu}=\partial_{\mu} A_{\nu}-\partial_{\nu} A_{\mu}$ is the tensor of e.m. field and $A_{\nu}$ is the four-vector of the photon field. This field $A_{\nu}$ is massive and so the gauge invariance is lost.

The breaking of gauge invariance caused by presence of massive term $m_{\gamma}^{2}$ has been tested with geomagnetic and galactic magnetic data. As a result, the limits for parameter $m_{\gamma}^{2}$ have been obtained in the form $m_{\gamma} \leq 3 \cdot 10^{-24}$ and $m_{\gamma} \leq 3 \cdot 10^{-36}$, correspondingly.

The other modification of the Maxwell Lagrangian was proposed by Carroll, Field and Jackiw:

$$
\mathcal{L}_{E M} \rightarrow L=\mathcal{L}_{E M}+\mathcal{L}_{C S}
$$

where $\mathcal{L}_{C S}$ is a four-dimensional version of the ChernSimons term:

$$
\mathcal{L}_{C S}=\frac{1}{4} \varepsilon_{\mu \nu \rho \sigma} F^{\mu} A^{\nu} F^{\rho \sigma} .
$$

Here $p_{\mu}$ is a constant vector which causes violation of the Lorentz-invariance.

The CFJ model presents a rather elegant and convenient way for testing possible violation of the Lorentz-invariance, which causes its large impact. But this model has a principle disadvantage, namely, the breaking of the Lorentz-invariance "by hands" and the additional constants $p_{\mu}$ have no physical meaning. In addition, this model is invariant with respect to neither the Lorentz nor Poincaré group, i.e. it does not satisfy any relativity principle accepted in physics.

In the following sections we discuss two dynamical versions of the CFJ model which contain the ordinary CFJ model as a particular sector. One of them is very similar to axion electrodynamics in which, however, the axion rest mass is zero. The other includes axion electrodynamics as a limiting case corresponding to a small coupling constant.

\section{The MCS model in the $(1+3)$-dimensional Minkovski space}

Let us start with the following Lagrangian

$$
\begin{aligned}
L= & -\frac{1}{4} F_{\mu \nu} F^{\mu \nu}-\frac{1}{2} F_{\mu} F^{\mu}- \\
& \frac{\kappa}{4} \varepsilon_{\mu \nu \rho \sigma} F^{\mu} A^{\nu} F^{\rho \sigma}+e j_{\mu} A^{\mu}+q j_{4} A^{4} .
\end{aligned}
$$

Here the Greek indices run over the values $0,1,2,3$, $A^{\nu}$ and $F^{\mu \nu}=\partial^{\mu} A^{\nu}-\partial^{\nu} A^{\mu}$ are the four-vector potential and tensor of the electromagnetic field, respectively. In addition, Lagrangian (3) includes a scalar 
potential $A^{4}$ and its derivatives $F^{\mu}=\frac{\partial A^{4}}{\partial x_{\mu}}$ as well as a scalar current $j_{4}$.

The Lagrangian (3) has the following nice properties.

- It is transparently invariant w.r.t. Lorentz transformations and shifts of independent variables. Moreover, since $j^{4}$ is a scalar, it is possible to introduce an additional charge $q$ which is not necessarily equal to the electric charge $e$ but in general to a coupling constant corresponding to some interaction which is not necessary purely electromagnetic.

- The corresponding energy-momenta tensor does not depend on parameter $\kappa$ and so is not affected by the term $-\frac{\kappa}{4} \varepsilon_{\mu \nu \rho \sigma} F^{\mu} A^{\nu} F^{\rho \sigma}$. More precisely, this tensor has the following form

$$
\begin{aligned}
T^{00} & =\frac{1}{2}\left(\mathbf{E}^{2}+\mathbf{B}^{2}+F_{0}^{2}+\mathbf{F}^{2}\right), \\
T^{0 a} & =\frac{1}{2}\left(\varepsilon_{a b c} E_{b} B_{c}+F^{0} F^{a}\right),
\end{aligned}
$$

where $\mathbf{E}, \mathbf{B}$ and $\mathbf{F}$ are three-vectors whose components are: $E_{a}=F_{0 a}, B_{a}=\frac{1}{2} \varepsilon_{a b c} F_{b c}$ and $F_{a}$.

- Lagrangian (3) includes both the field components $F^{\mu \nu}, F^{\nu}$ and potentials $A^{\mu}$. But in spite of the explicit dependence on potentials, the Lagrangian admits gauge transformations $A^{\mu} \rightarrow A^{\mu}+\partial^{\mu} \varphi$ since via them it is changed only by a mere surface term:

$$
L \rightarrow L+\partial^{\mu}\left(\varphi \varepsilon_{\mu \nu \rho \sigma} F^{\nu \rho} F^{\sigma}\right) .
$$

In addition, this Lagrangian is not affected by the change $A^{4} \rightarrow A^{4}+C$, where $C$ is a constant.

- In non-relativistic approximation, Lagrangian (3) is reduced to the Galilei-invariant Lagrangian for the irreducible Galilean field discussed in [3].

\section{$3 \quad$ Field equations}

Let us consider the Euler-Lagrange equations which correspond to Lagrangian (3):

$$
\begin{aligned}
\partial_{\nu} F^{\mu \nu}+\kappa F_{\nu} \widetilde{F}^{\mu \nu} & =e j^{\mu}, \\
\partial_{\nu} F^{\nu}+\frac{\kappa}{2} F_{\lambda \nu} \widetilde{F}^{\lambda \nu} & =q j^{4} .
\end{aligned}
$$

Here, $\widetilde{F}^{\mu \nu}=\frac{1}{2} \varepsilon^{\mu \nu \rho \sigma} F_{\rho \sigma}$ is the dual tensor of the electromagnetic field $F^{\mu \nu}$. Field variables $\widetilde{F}^{\mu \nu}$ and $F_{\mu}$ satisfy the following conditions:

$$
\partial_{\nu} \widetilde{F}^{\mu \nu}=0, \quad \partial_{\nu} F_{\mu}-\partial_{\mu} F_{\nu}=0
$$

in accordance with their definitions as derivatives of the potential.
Equations (5)-(7) involve ten field variables, i.e., six-component tensor $F^{\mu \nu}$ and four-vector $F^{\mu}$. All these variables are dynamical and of equal value. If $q=0$ then equations (5)-(7) reduce to the field equations of an axion electrodynamics [9] with zero axion mass.

First, let us note that equations (5)-(7) cover the system of equations proposed by Carroll, Field and Jackiw [2]. Indeed, the system (5)-(7) is compatible with the following additional condition

$$
\partial_{\nu} F_{\mu}=0 .
$$

If the condition (8) is imposed then $F_{\mu}=p_{\mu}$ where $p_{\mu}$ are constants. Substituting this solution into (5) we obtain the CFJ equations. Concerning our additional equation (6), for constant $p_{\mu}$ it is reduced to a definition of $j^{4}$.

Note that equations (5) with variable (i.e., nonconstant) $p_{\mu}$ were discussed in [7] in frames of the premetric approach [8]. However, $F^{\mu}$ is treated there as an external axion field, while in our model it is a dynamical variable satisfying evolution equation (6) and constraints (7).

\section{MCS model with nonliner Bianchy indentity}

The system (5), (6) generates the tensor conserved current whose components are given in equation (4). Moreover, the energy-momentum tensor (4) has a very simple form and does not depend on the coupling constant $\kappa$. On the other hand, for $\kappa=0$ this tensor can be segregated to two parts, each of which is conserved separately:

$$
T^{\mu \nu}=T_{1}^{\mu \nu}+T_{2}^{\mu \nu},
$$

where

$$
\begin{aligned}
& T_{1}^{00}=\frac{1}{2}\left(\mathbf{E}^{2}+\mathbf{B}^{2}\right), T_{1}^{0 a}=\frac{1}{2} \varepsilon_{a b c} E_{b} B_{c}, \\
& T_{2}^{00}=F_{0}^{2}+\mathbf{F}^{2}, T_{2}^{0 a}=\frac{1}{2} F^{0} F^{a} .
\end{aligned}
$$

However, for $\kappa$ nonzero either tensor (10) or (11) is not conserved, but only their sum (9) is a conserved quantity.

In this section we propose an MCS model which causes conservation of the standard energy-momenta tensor of Maxwell field given by expressions (10). The related field equations for antisymmetric tensor $F^{\mu \nu}$ are:

$$
\begin{aligned}
\partial_{\nu} F^{\mu \nu}+\kappa F_{\nu} \widetilde{F}^{\mu \nu} & =e j^{\mu}, \\
\partial_{\nu} \widetilde{F}^{\mu \nu}-\kappa F_{\nu} F^{\mu \nu} & =0
\end{aligned}
$$

where $F^{\mu}=\partial_{\mu} A^{4}$ and $A^{4}$ is a scalar potential satisfying the following nonlinear equation

$$
\begin{aligned}
\partial_{\nu} \partial^{\nu} A^{4}= & \kappa\left(F_{\mu \nu} F^{\mu \nu} \sin \left(\kappa A^{4}\right)-\right. \\
& \left.F_{\mu \nu} \widetilde{F}^{\mu \nu} \cos \left(\kappa A^{4}\right)\right) .
\end{aligned}
$$


It is easy to verify that tensor (10) satisfies the continuity equation $\partial_{\mu} T_{1}^{\mu \nu}=0$ provided $F^{\mu \nu}$ solve the field equations (12)-(13) with zero current $j^{\mu}=0$.

Like (5)-(7), equations (12)-(14) admit Lagrangian formulation. To construct the related Lagrangian we express $F^{\mu \nu}$ via potential $A=$ $\left(A_{0}, A_{1}, A_{2}, A_{3}, A_{4}\right)$ in a non-linear fashion:

$$
\begin{aligned}
F^{\mu \nu}= & \left(\partial^{\mu} A^{\nu}-\partial^{\nu} A^{\mu}\right) \cos \left(\kappa A^{4}\right)- \\
& \frac{1}{2} \varepsilon_{\lambda \sigma}^{\mu \nu}\left(\partial^{\lambda} A^{\sigma}-\partial^{\sigma} A^{\lambda}\right) \sin \left(\kappa A^{4}\right) .
\end{aligned}
$$

The Ansatz (15) converts equation (13) to identity, which plays the role of Bianchi identity in our model. Note that this identity appears to be essentially nonlinear.

Using definition (15), we can write a Lagrangian for the system (12)-(14):

$$
\begin{aligned}
L= & \frac{1}{4}\left(F_{\mu \nu} F^{\mu \nu} \cos \left(\kappa A^{4}\right)+\right. \\
& \left.F_{\mu \nu} \widetilde{F}^{\mu \nu} \sin \left(\kappa A^{4}\right)\right)+\frac{1}{2} F_{\mu} F^{\mu} .
\end{aligned}
$$

Variating (16) w.r.t. $A^{\mu}$ and $A^{4}$ one obtains equations (12) and (14) correspondingly.

For small values of parameter $\kappa$ and bounded $A^{4}$ it is possible to expand Lagrangian (16) and the related equations (12)-(14) in power series of $\kappa$. Then neglecting terms whose order in $\kappa$ is higher than one we obtain Lagrangian (3). In other words, the model considered in Sections 4 and 5 can be treated as a first approximation of the model based on Lagrangian (16).

\section{Continuous and discrete symmetries}

Equations (5)-(7) (or (24)) are transparently invariant w.r.t. the Poincaré group. Nevertheless we examined them using tools of Lie analysis and found their maximal invariance group. We will not present here details of this routine procedure, whose algorithm can be found in [10], but will formulate its result: equations (5)-(7) are invariant w.r.t. 11-parametrical extended Poincaré group $\widetilde{P}(1,3)$, whose infinitesimal generators are

$$
\begin{aligned}
& P_{\mu}=\partial_{\mu}, \quad J_{\mu \nu}=x_{\mu} \partial_{\nu}-x_{\nu} \partial_{\mu}+S_{\mu \nu}, \\
& D=x_{\mu} \partial^{\mu}-I-j_{\mu} \partial_{j_{\mu}} .
\end{aligned}
$$

Here $I=F_{\mu} \partial_{F_{\mu}}+F_{\mu \nu} \partial_{F_{\mu \nu}}$ is an operator which acts on the field variables as the unit one, $S_{\mu \nu}$ are generators of the Lorentz group acting on the field variables and currents:

$$
\begin{aligned}
S_{a b}= & K_{a b}-K_{b a}, \\
K_{a b}= & F_{a} \partial_{F_{b}}+E_{a} \partial_{E_{b}}+H_{a} \partial_{H_{b}}+j_{a} \partial_{j_{b}}, \\
& a, b \neq 0, \\
S_{0 a}= & F_{0} \partial_{F_{a}}+F_{a} \partial_{F_{0}}+ \\
& \varepsilon_{a b c}\left(E_{a} \partial_{H_{b}}-H_{a} \partial_{E_{b}} H_{b} \partial_{E_{a}}\right) .
\end{aligned}
$$

Thus, in contrast to the CFJ model, the considered CSM model in $(1+3)$-dimensional Minkovski space is invariant w.r.t. the extended Poincaré group. Note that the additional condition (8) also is invariant w.r.t. this group; violation of Lorentz invariance takes place only after fixing particular constant solutions $F_{\mu}=p_{\mu}$.

Equations (24) are also invariant w.r.t. discrete transformations of space reflection $P$ and time inversion $T$. Moreover, $F^{\mu}$ is a pseudovector and so the potential $A^{4}$ is a pseudoscalar.

In an analogous way we have found the maximal Lie group and discrete symmetries admitted by system (12)-(14). It happens that the symmetry of this system is completely analogous to the symmetry of equations (5)-(7). Namely, system (12)-(14) is invariant w.r.t. extended Poincaré group $\widetilde{P}(1,3)$, whose generators are given in equations (17), and admits discrete symmetry transformations $\mathrm{P}, \mathrm{C}$ and $\mathrm{T}$.

\section{Non-relativistic limit}

The correct definition of the non-relativistic limit is by no means a simple problem in general and in the case of theories of massless fields in particular, see, for example, [12]. A necessary (but not sufficient) condition for obtaining consistent non-relativistic limit of a relativistic theory is to take care that the limiting theory be in agreement with the principle of Galilean relativity [11].

To find a non-relativistic limit of equations (24) we use the Inönü-Wigner contraction [13], which guarantees Galilean symmetry of the limiting theory. Namely, we shall start with the representation of the Poincaré algebra, which is realized on the set of solutions of equations (24), and contract it to the representation of the Galilei algebra. Then, using the contraction matrix we find the Galilean limits of Lagrangian (3) and system (24).

The tensor field $F^{\mu \nu}$ and vector field $F^{\mu}$ transform in accordance with the representation

$$
[D(0,1) \oplus D(1,0)] \oplus D(1 / 2,1 / 2)
$$

of the Lorentz group. Contractions of this representation (and also of all irreducible representations involved into the direct sum (19)) to indecomposable representations of the homogeneous Galilei group $h g(1,3)$ were discussed in [14] and [15].

The contraction of (19) to the indecomposable representation of $h g(1,3)$ is reduced to the following procedure. First, let us represent the field variables as a ten component vector

$$
\begin{aligned}
\Psi= & \operatorname{column}\left(F^{01}, F^{02}, F^{03}, F^{23}, F^{31}, F^{12},\right. \\
& \left.F^{1}, F^{2}, F^{3}, F^{0}\right)
\end{aligned}
$$

then Lorentz generators (18) act on $\Psi$ as the following matrices 


$$
\begin{aligned}
& S_{a b}=\varepsilon_{a b c}\left(\begin{array}{cccc}
S_{c} & 0 & 0 & 0 \\
0 & S_{c} & 0 & 0 \\
0 & 0 & S_{c} & 0 \\
0 & 0 & 0 & 0
\end{array}\right) ; \\
& S_{0 a}=\left(\begin{array}{cccc}
0 & -S_{a} & 0 & 0 \\
S_{a} & 0 & 0 & 0 \\
0 & 0 & 0 & K_{a}^{\dagger} \\
0 & 0 & -K_{a} & 0
\end{array}\right) .
\end{aligned}
$$

Here $S_{a}$ are spin-one matrices whose elements are $\left(S_{a}\right)_{b c}=i \varepsilon_{a b c}$, and $K_{a}$ are $1 \times 3$ matrices of the form

$$
K_{1}=(\mathrm{i}, 0,0), \quad K_{2}=(0, \mathrm{i}, 0), \quad K_{3}=(0,0, \mathrm{i}),
$$

and 0 denote the zero matrices of an appropriate dimension.

The Inönü-Wigner contraction consists of transformation to a new basis $S_{a b} \rightarrow S_{a b}, S_{0 a} \rightarrow \varepsilon S_{0 a}$ followed by a similarity transformation of basis elements $S_{\mu \nu} \rightarrow S_{\mu \nu}^{\prime}=V S_{\mu \nu} V^{-1}$ with a matrix $V$ depending on contracting parameter $\varepsilon$. Moreover, $V$ should depend on $\varepsilon$ in a tricky way, such that all the transformed generators $S_{a b}^{\prime}$ and $\varepsilon S_{0 a}^{\prime}$ are kept non-trivial and non-singular when $\varepsilon \rightarrow 0$ [13].

In accordance with $[14,15]$, representation (19) can be contracted either to the indecomposable representation of $h g(1,3)$ or to a direct sum of such representations. To obtain an indecomposable representation, contraction matrix $V$ has to be chosen in the following form:

$$
\begin{aligned}
V & =\left(\begin{array}{cccc}
\frac{\varepsilon}{2} & 0 & \frac{\varepsilon}{2} & 0 \\
0 & I & 0 & 0 \\
-\varepsilon^{-1} & 0 & \varepsilon^{-1} & 0 \\
0 & 0 & 0 & 1
\end{array}\right), \\
V^{-1} & =\left(\begin{array}{cccc}
\varepsilon^{-1} & 0 & -\frac{\varepsilon}{2} & 0 \\
0 & I & 0 & 0 \\
\varepsilon^{-1} & 0 & \frac{\varepsilon}{2} & 0 \\
0 & 0 & 0 & 1
\end{array}\right) .
\end{aligned}
$$

To apply the contraction procedure to the field equations (5)-(7) we first write them in vector notations

$$
\begin{aligned}
\partial_{0} \mathbf{E}-\nabla \times \mathbf{B} & =\kappa\left(F_{0} \mathbf{B}-\mathbf{F} \times \mathbf{E}\right)+e \mathbf{j}, \\
\nabla \cdot \mathbf{E} & =\kappa \mathbf{F} \cdot \mathbf{B}+e j^{0}, \\
\partial_{0} \mathbf{B}+\nabla \times \mathbf{E} & =0 \\
\nabla \cdot \mathbf{B} & =0 \\
\partial_{0} F_{0}-\nabla \cdot \mathbf{F} & =-\kappa \mathbf{E} \cdot \mathbf{B}+q j^{4} \\
\partial_{0} \mathbf{F}-\nabla F_{0} & =0 \\
\nabla \times \mathbf{F} & =0
\end{aligned}
$$

Taking half sums and half divergences of pairs of equation (25) and (28), (24) and (29), (26) and (30) we come to a system equivalent to $(24)-(30)$ :

$$
\begin{aligned}
& \partial_{0} F_{0}-\nabla \cdot(\mathbf{F}-\mathbf{E})=\kappa(\mathbf{F}-\mathbf{E}) \cdot \mathbf{B}+e\left(j^{0}+\frac{q}{e} j^{4}\right), \\
& \partial_{0} F_{0}-\nabla \cdot(\mathbf{F}+\mathbf{E})=\kappa(\mathbf{F}+\mathbf{E}) \cdot \mathbf{B}+e\left(j^{0}-\frac{q}{e} j^{4}\right), \\
& \partial_{0}(\mathbf{E}+\mathbf{F})-\nabla \times \mathbf{B}-\nabla F_{0}= \\
& \kappa\left(F_{0} \mathbf{B}-\frac{1}{2}(\mathbf{F}-\mathbf{E}) \times(\mathbf{F}+\mathbf{E})\right)+e \mathbf{j}, \\
& \partial_{0}(\mathbf{E}-\mathbf{F})-\nabla \times \mathbf{B}+\nabla F_{0}= \\
& \kappa\left(F_{0} \mathbf{B}-\frac{1}{2}(\mathbf{F}-\mathbf{E}) \times(\mathbf{F}+\mathbf{E})\right)+e \mathbf{j}, \\
& \partial_{0} \mathbf{B}+\nabla \times(\mathbf{E}+\mathbf{F})=0, \\
& \partial_{0} \mathbf{B}+\nabla \times(\mathbf{E}-\mathbf{F})=0, \nabla \cdot \mathbf{B}=0 .
\end{aligned}
$$

Defining $\Psi^{\prime}=\operatorname{column}\left(\mathbf{F}^{\prime}, \mathbf{B}^{\prime}, \mathbf{E}^{\prime}, F_{0}^{\prime}\right)=V \Psi$ we obtain from $(20),(23)$ :

$$
\begin{aligned}
& \mathbf{E}+\mathbf{F}=2 \varepsilon^{-1} \mathbf{F}^{\prime}, \mathbf{B}^{\prime}=\mathbf{B}, \\
& \mathbf{F}-\mathbf{E}=\varepsilon \mathbf{F}^{\prime}, F_{0}=F_{0}^{\prime} \\
& 2 \varepsilon^{-1} j^{4}=\left(\frac{q}{e} j^{4}-j^{0}\right), \\
& \varepsilon j^{\prime 0}=\frac{q}{e} j^{4}+j^{0}, \mathbf{j}^{\prime}=\mathbf{j} .
\end{aligned}
$$

Substituting (32) into (31), equating terms with lowest powers of $\varepsilon$ and taking into account that relativistic variable $x_{0}$ is related to non-relativistic time $t$ as $x_{0}=c t=\varepsilon^{-1} t$ we obtain the following system:

$$
\begin{aligned}
& \partial_{t} F_{0}^{\prime}-\nabla \cdot \mathbf{E}^{\prime}+\kappa \mathbf{B}^{\prime} \cdot \mathbf{E}^{\prime}=e j^{\prime 0}, \\
& \partial_{t} \mathbf{F}^{\prime}+\nabla \times \mathbf{B}^{\prime}+\kappa\left(F_{0}^{\prime} \mathbf{B}^{\prime}+\mathbf{F}^{\prime} \times \mathbf{E}^{\prime}\right)=e \mathbf{j}^{\prime}, \\
& \nabla \cdot \mathbf{F}^{\prime}+\kappa \mathbf{F}^{\prime} \cdot \mathbf{B}^{\prime}=e j^{\prime 4}, \\
& \partial_{t} \mathbf{B}^{\prime}+\nabla \times \mathbf{E}^{\prime}=0, \nabla \cdot \mathbf{B}^{\prime}=0, \\
& \nabla \times \mathbf{F}^{\prime}=0, \partial_{t} \mathbf{F}^{\prime}=\nabla{F^{\prime}}_{0} .
\end{aligned}
$$

System of equations (33) coincides with the Galilei invariant system for the indecomposable ten component field deduced in [3]. Like the corresponding relativistic equations (5), (6), system (33) admits a Lagrangian formulation. The related Lagrangian can be obtained from (3) using the contraction procedure and has the following form

$$
\begin{aligned}
L= & \frac{1}{2}\left({F_{0}^{\prime}}^{2}-\mathbf{B}^{2}\right)-\mathbf{E}^{\prime} \cdot \mathbf{F}^{\prime}+ \\
& \kappa\left(A^{0} \mathbf{B}^{\prime} \cdot \mathbf{F}^{\prime}-\mathbf{A}^{\prime} \cdot\left(\mathbf{B}^{\prime} F_{0}^{\prime}+\mathbf{F}^{\prime} \times \mathbf{E}^{\prime}\right)\right)-(34) \\
& e\left(A^{\prime 0} j^{\prime 4}+A^{\prime 4} j^{\prime 0}-\mathbf{j}^{\prime} \cdot \mathbf{A}^{\prime}\right) .
\end{aligned}
$$

Just the system (33) and Lagrangian (34) represent the Galilean limit of the model discussed in Sections 4 and 5. Exactly this lagrangian was found in [3] starting with the Galilei invariance condition. 
With the additional Galilei-invariant constraints

$$
\mathbf{F}^{\prime}=0, \quad j^{\prime 4}=0, \quad F_{0}^{\prime}=p_{0}=\mathrm{const}
$$

system (33) is reduced to the form

$$
\begin{aligned}
& \frac{\partial \mathbf{B}^{\prime}}{\partial t}+\nabla \times \mathbf{E}^{\prime}=0, \\
& \nabla \cdot \mathbf{B}^{\prime}=0, \\
& \nabla \cdot \mathbf{E}^{\prime}+\kappa \mathbf{B}^{\prime} \cdot \mathbf{E}^{\prime}=e j^{\prime 0}, \\
& \nabla \times \mathbf{B}^{\prime}+\kappa p_{0} \mathbf{B}^{\prime}=e \mathbf{j}^{\prime} .
\end{aligned}
$$

Equations (35) represent a Galilei-invariant version of the CFJ model with time-like $p_{\mu}$.

\section{$7 \quad$ Exact solutions}

One of important applications of Lie symmetries to partial differential equations (PDE) is connected with constructing their exact solutions.

Lie algorithm for constructing exact solutions of differential equations has been known for more than 120 years, see, e.g., [10]. Various applications of this algorithm to relativistic systems can be found in [16].

In this section we present some group solutions of the relativistic system (5)-(7). Since the maximal Lie symmetry of this system has been found and presented in Section 6, it is possible to find its exact solutions using the following algorithm:

1. To find all non-equivalent three-dimensional subalgebras of the Lie algebra of group $\tilde{P}(1,3)$ whose generators are given by formulae (17).

2 . To find invariants of the related threeparametric Lie groups.

3. To choose new variables in such a way that eight of them coincides with these invariants. As a result we obtain a system of ordinary differential equations.

4. To solve (if possible) the obtained systems of ordinary differential equations and reconstruct the related solutions of the incoming system.

The first step of the algorithm is reduced to using the classification results for the subalgebras of algebra $P(1,3)$, which can be found in [17]. All the remaining steps are rather cumbersome but algorithmic, and it is possible to find all exact solutions for systems (5)-(7), (12)-(14) which can be obtained via Lie reduction procedure. Here we shall present only two examples of such solutions, while the complete list of them can be found in [18].

Let us start with the subalgebra of $\tilde{p}(1,3)$, spanned on the basis elements $<J_{12}, P_{1}, P_{2}>$ which are given explicitly by equations (17) and (18). There is the only invariant of the related group depending on $x_{0}, x_{1}, x_{2}$ and $x_{3}$, namely, $\omega=x_{1}^{2}+x_{2}^{2}$. In addition, there are seven invariants depending on both space-time and field variables, which we denote as $\varphi_{1}, \varphi_{2}, \cdots, \varphi_{7}$. They are supposed to be functions of $\omega$ such that

$$
\begin{aligned}
& B_{1}=\varphi_{1} \cos \omega-\varphi_{3} \sin \omega, \\
& B_{2}=\varphi_{1} \sin \omega+\varphi_{2} \cos \omega, \\
& E_{1}=\varphi_{3} \cos \omega-\varphi_{4} \sin \omega, \\
& E_{2}=\varphi_{3} \sin \omega+\varphi_{4} \cos \omega, \\
& B_{3}=\varphi_{5}, E_{3}=\varphi_{6}, A^{4}=\varphi_{7} .
\end{aligned}
$$

Substituting (36) into (5)-(7) we reduce it to a system of ordinary differential equations which appears to be integrable. Moreover, its general solution depends on six arbitrary parameters. A particular solution has the following form:

$$
\begin{aligned}
& B_{1}=-c_{1} x_{2} / \omega, \quad B_{2}=c_{1} x_{1} / \omega, \quad B_{3}=c_{3} A^{4}, \\
& E_{1}=c_{2} x_{1} / \omega, \quad E_{2}=c_{2} x_{2} / \omega, \quad E_{3}=c_{3}, \\
& F_{0}=F_{3}=0, \quad F_{\alpha}=\nabla_{\alpha} A^{4}, \quad \alpha=1,2
\end{aligned}
$$

where $A^{4}=c_{4} J_{0}\left(c_{3} \sqrt{k \omega}\right)+c_{5} Y_{0}\left(c_{3} \sqrt{k \omega}\right), J_{0}$ and $Y_{0}$ are the Bessel functions of the first and second kind, respectively, $c_{1}, \cdots c_{5}$ are arbitrary parameters.

It is interesting to note that functions (37) and (38) also solve the standard (linear) Maxwell equations with bounded currents. Namely, the electric field (38) coincides with the field of an infinite straight charged line coinciding with the third coordinate axis supplemented by the constant electric field $E_{3}=c_{3}$. The magnetic field (37) is a superposition of the field of a straight line current directed along the third coordinate axis and the field $E_{3}=c_{3} A_{4}$ generated by the current whose components are $j_{1}=-\frac{x_{2}}{\sqrt{\omega}} A_{4}^{\prime}, j_{2}=$ $\frac{x_{1}}{\sqrt{\omega}} A_{4}^{\prime}, j_{3}=0$, where $A_{4}^{\prime}=\frac{\partial A_{4}}{\partial \omega}$.

Let us present one more exact solution of the system $(5)-(7)$ :

$$
\begin{aligned}
& B_{1}=c \frac{x_{2}}{\omega^{3 / 2}}, B_{2}=c \frac{-x_{1}}{\omega^{3 / 2}}, B_{3}=0, \\
& E_{1}=c \frac{x_{1}}{\omega^{3 / 2}}, E_{2}=c \frac{x_{2}}{\omega^{3 / 2}}, E_{3}=0, \\
& F_{1}=\frac{x_{2}}{\omega}, F_{2}=-\frac{x_{1}}{\omega}, F_{3}=F_{0}=0, \\
& j_{\mu}=0, \mu=0, \cdots, 4
\end{aligned}
$$

In contrast with (37)-(39) vectors $\mathbf{B}$ and $\mathbf{E}$ defined in (40) do not solve linear Maxwell equations with bounded currents. However, they solve the system of nonlinear equations (5)-(7) for $\omega>0$.

A complete set of reductions and exact solutions for equations (5)-(7) can be found in [20].

\section{Conclusion}

We have discused two MCS models in the $(1+3)$ dimensional space-time. One of them is presented in Sections 2 and 3. It generalizes the axion electrodynamics to a theory with a five-component current. The other model includes axion electrodynamics as a limiting case corresponding to a small coupling constant. This model includes a non-linear version of the Bianchi 
identity. The other specific feature of this model is the existence of two conserved parts of the energy density, one of which corresponds to the tensor of the electromagnetic field while the other one is formed by the additional four-vector field $F^{\mu}$.

In contrast to the CFJ model, our models are relativistically invariant and include this model as a particular sector corresponding to constant solutions for vector field $F^{\mu}$. Both the models have good nonrelativistic limit, coinciding with the Galilei invariant system discussed in [3]. To obtain this limit we apply the Inönü-Wigner contraction, which makes it possible to find a Galilean formulation of the CFJ model.

Using the classical Lie approach, we find continuous symmetries of our models and construct multiparameter families of their exact solutions. Two of these solutions are presented in Section 7, while the complete list of group solutions can be found in preprint [18].

Note that solutions (37)-(39) and (40) give rise to new exactly solvable models for Dirac fermions. One of such models is presented in [19].

\section{References}

[1] Snonfeld, J.: Nucl. Phys., B175 157, 1991; Deser, S., Jackiw, R., Templeton, S.: Ann. Phys. $140372,1982$.

[2] Carrol, S. M., Field, J. B., Jackiw, R.: Phys. Rev. D 41 1231, 1990.

[3] Niederle, J., Nikitin, A. G.: J. Phys. A: Mat. Theor. 42 105207, 2009.

[4] Chern, S. S., Simons, J.: Annals Math. 99 48, 1974.

[5] Horvaty, P. A.: Lections on (Abelian) ChernSimons vortices. ArXiv 0704.3220

[6] Hariton, A. J., Legnert, R.: Phys. Lett. A 367 11, 2007.

[7] Itin, Ya.: Phys. Rev. D 70 025012, 2004; Itin, Ya.: Wave propagation in axion electrodynamics, ArXiv 0706.2991.

[8] Obukhov, Y. N., Hehl, F. W.: Phys. Lett. B 458, 466, 1999;

Obukhov, Y. N., Rubilar, G. F.: Phys. Rev. D 66, 2002.

[9] Wilczek, F.: Phys. Rev. Lett. 58, 1799, 1987.
[10] Olver, P.: Application of Lie groups to Differential equations. Springer-Verlag, N.Y., 1986.

[11] Le Bellac, M., Lévy-Leblond, J.-M.: Nuovo Cimento B 14, 217, 1973.

[12] Holland, P., Brown, H. R.: Studies in History and Philosophy of Science 34, 161, 2003.

[13] Inönü, E., Wigner, E. P.: Proc. Nat. Acad. Sci. U.S. 39, 510, 1953.

[14] de Montigny, M., Niederle, J., Nikitin, A. G.: J. Phys. A: Mat. Theor. 39, 1, 2006.

[15] Niederle, J., Nikitin, A. G.: Czech. J. Phys. 56, $1243,2006$.

[16] Fushchich, W. I., Nikitin, A. G.: Symmetries of Equations of Quantum Mechanics. New York, Allerton Press, 1994.

[17] Fushchich, W. I., Barannyk, A. F., Barannyk, L. F.: Subgroup structure of Lie groups. Naukova Dumka, Kiev, 1993.

[18] Kuriksha, O., Nikitin, A. G.: Arxiv preprint arXiv:1002.0064, 2010.

[19] Ferraro, E., Messina, N., Nikitin, A. G.: Phys. Rev. A 81, 042108, 2010, ArXiv 0909.5543.

[20] Kuriksha, O.: Group analysis of $(1+3)$-dimensional Maxwell-Chern-Simons models and their exact solutions. ArXiv 0911.3220.

J. Niederle

E-mail: niederle@fzu.cz

Institute of Physics of the

Academy of Sciences of the Czech Republic

Na Slovance 2, 18221 Prague, Czech Republic

A. G. Nikitin

E-mail: nikitin@imath.kiev.ua

Institute of Mathematics

National Academy of Sciences of Ukraine

3 Tereshchenkivs'ka Street, Kyiv-4, Ukraine, 01601

O. Kuriksha

Institute of Mathematics

National Academy of Sciences of Ukraine

3 Tereshchenkivs'ka Street, Kyiv-4, Ukraine, 01601 